PROCEEDINGS OF THE

AMERICAN MATHEMATICAL SOCIETY

Volume 131, Number 7, Pages 2071-2079

S 0002-9939(03)07089-8

Article electronically published on February 13, 2003

\title{
COMMENTS ABOUT THE STEINHAUS TILING PROBLEM
}

\author{
R. DANIEL MAULDIN AND ANDREW Q. YINGST
}

(Communicated by David Preiss)

\begin{abstract}
Recently, using Fourier transform methods, it was shown that there is no measurable Steinhaus set in $\mathbb{R}^{3}$, a set which no matter how translated and rotated contains exactly one integer lattice point. Here, we show that this argument cannot generalize to any lattice and, on the other hand, give some lattices to which this method applies. We also show there is no measurable Steinhaus set for a special honeycomb lattice, the standard tetrahedral lattice in $\mathbb{R}^{3}$.
\end{abstract}

\section{INTRODUCTION}

The Steinhaus tiling problem, first proposed by Steinhaus in 1957, is whether there exists a set in the plane which, under any isometry, contains exactly one point of $\mathbb{Z}^{2}$. Recently, Jackson and Mauldin [4] have constructed such a set. The question of whether such a subset of $\mathbb{R}^{2}$ can be measurable remains open although there are several partial results [1, [2], [5]; in [5] it is shown that such a set cannot have the Baire property.

Kolountzakis and Papadimitrakis 3 considered a variation of this problem: Does there exist a measurable subset $E$ of $\mathbb{R}^{d}$ such that for almost every $x \in \mathbb{R}^{d}$ and almost every isometry $S$, the set $(S E+x)$ contains exactly one point of $\mathbb{Z}^{d}$ ? They showed the answer to this question is no, for $d \geq 3$. This result had been shown earlier by Kolountzakis and Wolff [5] by more complicated means which also yield some stronger results. One purpose of this note is to examine how far the argument given in [3] might extend. We begin by repeating the key aspect of this argument, by generalizing from the lattice $\mathbb{Z}^{d}$, for $d \geq 3$, to the lattice $B \mathbb{Z}^{d}$, where $B \in G L(d, \mathbb{R})$.

We call the above condition the "almost sure" Steinhaus property on B or on the lattice $B \mathbb{Z}^{d}$. Specifically, a set $E$ is said to have the almost sure Steinhaus property on $B$ or on the lattice $B \mathbb{Z}^{d}$, where $B$ is an invertible matrix, provided that under almost every isometry $S$ and almost every point $x,\left|(S E+x) \cap\left(B \mathbb{Z}^{3}\right)\right|=1$. For the remainder of the paper we shall suppress the words almost sure. Observe that this property may be described as follows:

$$
\sum_{n \in B \mathbb{Z}^{d}} \mathbf{1}_{S E}(x-n)=1, \quad \text { a.e. } x \in \mathbb{R}^{d} \text {, a.e. isometry } S .
$$

Received by the editors October 15, 2001.

2000 Mathematics Subject Classification. Primary 28A20; Secondary 11K31, 60F20.

Key words and phrases. Method of descent, Fourier transform, lattice, quadratic form.

Both authors were supported in part by NSF Grant DMS 0100078. 
Let $\Lambda_{A}=A \mathbb{Z}^{d} \subset \mathbb{R}^{d}$, for $A \in G L(d, \mathbb{R})$, be the lattice induced by $A$, and let $\Lambda_{A}^{*}=A^{-T} \mathbb{Z}^{d}$ be its dual lattice. From elementary harmonic analysis, we have that if $f$ is an $L^{1}$ function, then

$$
\sum_{\lambda \in \Lambda_{A}} f(x-\lambda)=C, \quad \text { a.e. } x,
$$

if and only if its Fourier transform satisfies:

$$
\hat{f}(\lambda)=0, \quad \forall \lambda: \lambda \in \Lambda_{A}^{*} \backslash\{0\} .
$$

By integrating both sides of (2) over the parallelepiped spanned by the columns of $A$, we find that the constant $C$ equals the integral of $f$ times $\left|\operatorname{det}\left(A^{-1}\right)\right|$.

It follows from this that a measurable set $E$ has the almost sure Steinhaus property on $A$ if and only if $\mu(E)=|\operatorname{det}(A)|$, where $\mu(E)$ is the Lebesgue measure of $E$, and $\widehat{\mathbf{1}_{E}}$ vanishes on all points $x$, such that $\|x\|=\|\lambda\|$ for some $\lambda \in \Lambda_{A}^{*}, \lambda \neq 0$. In view of this, given a matrix $M$, let $\mathcal{D}(M)=\left\{\|M x\|^{2} \mid x \in \mathbb{Z}^{d}\right\}$ be the set of possible square distances between points of the lattice $M \mathbb{Z}^{d}$.

We are now in a position to give sufficient conditions under which there is no measurable set with the almost sure Steinhaus property on $B$. To this end, suppose we can find a matrix $A$ such that $\mathcal{D}\left(A^{-T}\right) \subseteq \mathcal{D}\left(B^{-T}\right)$, and such that $\operatorname{det}\left(A^{-T}\right) / \operatorname{det}\left(B^{-T}\right)=\operatorname{det}(B) / \operatorname{det}(A)$ is not an integer. Now suppose, by way of contradiction, that a measurable set $E$ has the Steinhaus property on $B$. Then $\widehat{\mathbf{1}_{E}}$ vanishes on all nonzero points with norm square in $\mathcal{D}\left(B^{-T}\right)$. So, $\widehat{\mathbf{1}_{E}}$ vanishes on all nonzero points with norm square in $\mathcal{D}\left(A^{-T}\right)$. This means $\widehat{\mathbf{1}_{E}}$ vanishes on $\Lambda_{A}^{*} \backslash\{0\}$. This gives us

$$
\sum_{\lambda \in \Lambda_{A}} \mathbf{1}_{E}(x-\lambda)=\frac{|\operatorname{det}(B)|}{|\operatorname{det}(A)|}, \quad \text { a.e. } x .
$$

It is easy to see that the left side must be an integer, and we have supposed that the right side is not. We adopt a notation with which to state this result.

If $A$ and $B$ are matrices such that $\mathcal{D}(A) \subseteq \mathcal{D}(B)$, we say $B$ norm dominates $A$, and write $B \succ A$ or $A \prec B$. If $B \succ A$ and we have that $\operatorname{det}(A) / \operatorname{det}(B)$ is irrational, we say $B$ strongly norm dominates $A$, and write $B \succ_{s} A$. If $B \succ A$ and we have $\operatorname{det}(A) / \operatorname{det}(B)$ not an integer, we say $B$ weakly norm dominates $A$, and write $B \succ_{w} A$. Finally, if $B \succ A$ and $\operatorname{det}(A) / \operatorname{det}(B) \in \mathbb{Z}$, we say $B$ trivially norm dominates $A$, and write $B \succ_{t} A$. With this terminology in place, we have proven the following theorem.

Theorem 1. Let $B \in G L(d, \mathbb{R})$ and suppose there exists a matrix $A \in G L(d, \mathbb{R})$, where $B^{-T} \succ_{w} A$. Then there is no measurable set with the almost sure Steinhaus property on $B$.

The remainder of this paper deals with the question of when a matrix $A$ exists such that $B \succ_{w} A$. To this end, it is useful to note the following two-part strategy: if we can find a matrix $C$ such that $C \succ_{t} B$ and $B \succ_{s} A$, then $C \succ_{s} A$ and, of course, $C \succ_{w} A$. Kouluntzakis and Papadimitrakis [3] have resolved some issues about this strategy for the case $B=I$. They show that in case $d=2$, there is no such $A$ so that this strategy cannot be applied. In case $d>2$, there is such an $A$ and so the strategy applies. We will not complete their proof here, but mention 
that, for $d=3$, their proof concludes by showing (under different terminology) that

$$
\left[\begin{array}{lll}
1 & & \\
& 1 & \\
& & 1
\end{array}\right] \succ_{w}\left[\begin{array}{ccc}
\sqrt{2} & & \\
& \sqrt{6} & \\
& & \sqrt{18}
\end{array}\right] .
$$

In section 2, we prove that if $A^{T} A$ has rational entries, then there is a diagonal matrix $B$ such that $A \succ_{t} B$ and $B^{T} B$ has rational entires. Thus, we can carry out the first part of the strategy. In section 3 , we demonstrate the limitation of the general method by exhibiting a class of diagonal matrices such that if $A \succ B$, then $A \succ_{t} B$. In section 4, we provide an example of a matrix $H$ for which the strategy works. Our proof uses some special quadratic forms and the method of descent.

\section{A RESUlt on NORM RATiOnal Matrices}

Let us refer to an invertible matrix $A$ as norm rational if $A^{T} A$ has only rational entries. This is equivalent to saying that the inner product of any two columns of $A$ is rational and also equivalent to saying that $\|A x\|^{2}$ is rational for every $x \in \mathbb{Z}^{d}$.

Theorem 2. Let $A \in G L(d, \mathbb{R})$ be norm rational. Then there is a diagonal norm rational matrix $B$, also in $G L(d, \mathbb{R})$, such that $A \succ_{t} B$.

Note that this theorem gives a halfway point toward showing that Theorem 1 applies to all norm rational matrices.

For the sake of clarity, we prove the theorem in the case of $d=3$, indicating how to generalize where appropriate.

Proof of Theorem 2. First, if $R$ is any linear isometry, then $B \succ A$ if and only if $R B \succ A$, since $\mathcal{D}(B)=\mathcal{D}(R B)$. Also, $A$ is norm rational if and only if $R A$ is norm rational. Therefore, we may assume $A$ is lower triangular and norm rational. Let us say

$$
A=\left[\begin{array}{ccc}
a_{1,1} & & \\
a_{2,1} & a_{2,2} & \\
a_{3,1} & a_{3,2} & a_{3,3}
\end{array}\right] .
$$

Our first goal is to show that general $a_{i, j}$ may be written as $q_{i, j} \sqrt{n_{i}}$, for some $q_{i, j} \in \mathbb{Q}, n_{i} \in \mathbb{Z}$. To do this, we note that any number $x$ with $x^{2} \in \mathbb{Q}$ may be written uniquely as $q \sqrt{n}$, where $q$ is rational and $n$ is a square free integer. This implies that if $x=q_{1} \sqrt{n} \neq 0$ and $x y \in \mathbb{Q}$, then $y=q_{2} \sqrt{n}$, for some rational $q_{2}$.

We denote the $i$ th column of $A$ as $A_{i}$. We have $\left\langle A_{3}, A_{3}\right\rangle=a_{3,3}^{2} \in \mathbb{Q}$, so we may write $a_{3,3}=q_{3,3} \sqrt{n_{3}}$. Next, we have $\left\langle A_{1}, A_{3}\right\rangle=a_{3,1} a_{3,3} \in \mathbb{Q}$ and $\left\langle A_{1}, A_{2}\right\rangle=a_{3,1} a_{3,2} \in \mathbb{Q}$, so by the second above property, we may also write $a_{3,1}=q_{3,1} \sqrt{n_{3}}, a_{3,2}=q_{3,2} \sqrt{n_{3}}$. This is valid since $\operatorname{det}(A) \neq 0$ implies $a_{3,3} \neq 0$. Note that this gives $a_{3, i} a_{3, j} \in \mathbb{Q}$ for all $i, j$.

We now proceed to row 2. We have $\left\langle A_{2}, A_{2}\right\rangle=a_{2,2}^{2}+a_{3,2}^{2} \in \mathbb{Q}$. Since we have $a_{3,2}^{2} \in \mathbb{Q}$, this gives $a_{2,2}^{2} \in \mathbb{Q}$, so we may write $a_{2,2}=q_{2,2} \sqrt{n_{2}}$. We have $\left\langle A_{1}, A_{2}\right\rangle=a_{2,1} a_{2,2}+a_{3,1} a_{3,2} \in \mathbb{Q}$, which gives $a_{2,1} a_{2,2} \in \mathbb{Q}$. Again, we have $a_{2,2} \neq 0$, so we may write $a_{2,1}=q_{2,1} \sqrt{n_{2}}$.

Finally, we have $\left\langle A_{1}, A_{1}\right\rangle=a_{1,1}^{2}+a_{1,2^{2}}+a_{1,3}^{2} \in \mathbb{Q}$, which gives $a_{1,1}^{2} \in \mathbb{Q}$, so we may write $a_{1,1}$ in the desired form.

The method for general dimension $d$ is similar to the above. We proceed row by row upwards, beginning with the diagonal element, which gives all other elements in the row. 
We let $v_{i}$ be a common denominator of the entries $q_{i, j}$ in row $i$, letting us write $q_{i, j}=\frac{u_{i, j}}{v_{i}}$, where $u_{i, j}$ and $v_{i}$ are integers. Now we examine

$$
\begin{aligned}
\mathcal{D}(A) & =\left\{\|A x\|^{2}: x \in \mathbb{Z}^{3}\right\} \\
& =\left\{\left(a_{1,1} x\right)^{2}+\left(a_{2,1} x+a_{2,2} y\right)^{2}+\left(a_{3,1} x+a_{3,2} y+a_{3,3} z\right)^{2}: \vec{x} \in \mathbb{Z}^{3}\right\} \\
& =\left\{\frac{n_{1}}{v_{1}^{2}}\left(a_{1,1} x\right)^{2}+\frac{n_{2}}{v_{2}^{2}}\left(a_{2,1} x+a_{2,2} y\right)^{2}+\frac{n_{3}}{v_{3}^{2}}\left(a_{3,1} x+a_{3,2} y+a_{3,3} z\right)^{2}: \vec{x} \in \mathbb{Z}^{3}\right\} .
\end{aligned}
$$

If $x_{1}, y_{1}$, and $z_{1}$ are nonzero integers, we can consider the subset of the above when $x_{1} \mid x$, and so on, and consider $x=x_{1} \hat{x}, y=y_{1} \hat{y} z=z_{1} \hat{z}$. The above continues as:

$$
\begin{aligned}
& \supseteq\left\{\frac{n_{1}}{v_{1}^{2}}\left(a_{1,1} x_{1} \hat{x}\right)^{2}+\frac{n_{2}}{v_{2}^{2}}\left(a_{2,1} x_{1} \hat{x}+a_{2,2} y_{1} \hat{y}\right)^{2}\right. \\
& \left.\quad+\frac{n_{3}}{v_{3}^{2}}\left(a_{3,1} x_{1} \hat{x}+a_{3,2} y_{1} \hat{y}+a_{3,3} z_{1} \hat{z}\right)^{2}: \overrightarrow{\hat{x}} \in \mathbb{Z}^{3}\right\} . \\
& =\left\{\frac{n_{1} a_{1,1}^{2} x_{1}^{2}}{v_{1}^{2}}(\hat{x})^{2}+\frac{n_{2} a_{2,2}^{2} y_{1}^{2}}{v_{2}^{2}}\left(\frac{a_{2,1} x_{1}}{a_{2,2} y_{1}} \hat{x}+\hat{y}\right)^{2}\right. \\
& \left.\quad+\frac{n_{3} a_{3,3}^{2} z_{1}^{2}}{v_{3}^{2}}\left(\frac{a_{3,1} x_{1}}{a_{3,3} z_{1}} \hat{x}+\frac{a_{3,2} y_{1}}{a_{3,3} z_{1}} \hat{y}+\hat{z}\right)^{2}: \overrightarrow{\hat{x}} \in \mathbb{Z}^{3}\right\} .
\end{aligned}
$$

Suppose nonzero integers $x_{1}, y_{1}$, and $z_{1}$ have been chosen such that $\frac{a_{2,1} x_{1}}{a_{2,2} y_{1}}, \frac{a_{3,1} x_{1}}{a_{3,3} z_{1}}$, and $\frac{a_{3,2} y_{1}}{a_{3,3} z_{1}}$ are integers. This is clearly possible; one method would be to let $z_{1}$ be one, then choose $y_{1}$ such that the third is an integer, and then choose $x_{1}$ so that the first two are integers. (In the higher dimensional case, we have $\frac{d(d-1)}{2}$ fractions which we wish to force to be integers. In that fraction which involves the $i$ th and $j$ th variable, with $i<j$, we will have the $i$ th variable on top, so that we may assign the parameters in decreasing order as in the case of $d=3$.) This allows a transformation which gives us

$$
=\left\{\frac{n_{1} a_{1,1}^{2} x_{1}^{2}}{v_{1}^{2}}(\hat{x})^{2}+\frac{n_{2} a_{2,2}^{2} y_{1}^{2}}{v_{2}^{2}}(\hat{y})^{2}+\frac{n_{3} a_{3,3}^{2} z_{1}^{2}}{v_{3}^{2}}(\hat{z})^{2}: \overrightarrow{\hat{x}} \in \mathbb{Z}^{3}\right\}=\mathcal{D}(B),
$$

where $B$ is defined by

$$
B=\mathcal{D}\left(\left[\begin{array}{lll}
\frac{a_{1,1} x_{1} \sqrt{n_{1}}}{v_{1}} & & \\
& \frac{a_{2,2} y_{1} \sqrt{n_{2}}}{v_{2}} & \\
& & \frac{a_{3,3} z_{1} \sqrt{n_{3}}}{v_{3}}
\end{array}\right]\right) .
$$

We have shown $A \succ B$, and it is easy to check that $\operatorname{det}(B) / \operatorname{det}(A)$ is $x_{1} y_{1} z_{1}$, and so is an integer, giving us $A \succ_{t} B$.

Here, we should clear up some possible confusion over the application of Theorem 1. Theorem 1 will give us that there is no measurable set with the Steinhaus property over $B$, if we have that $B^{-T}$ weakly norm dominates another matrix. For this reason, it is useful to note that $B$ is a norm rational matrix if and only if $B^{-T}$ is a norm rational matrix, since $B^{T} B$ is rational if and only if its inverse, $B^{-1} B^{-T}=\left(B^{-T}\right)^{T} B^{-T}$, is rational. This means that Theorem 2 is useful towards showing a matrix $B$ yields no set having the Steinhaus property on $B$ only if $B$ is norm rational. This will be examined further in the fourth section, where we will use this theorem to show that there is no Steinhaus set on the honeycomb lattice in $\mathbb{R}^{3}$. 


\section{A MATRIX WHICH WEAKLY NORM DOMINATES NO MATRIX}

Here, we show that the result or technique given in section 1 cannot always be used to show that there is no measurable set with the Steinhaus property over any $A$ in $\mathbb{R}^{3}$. For the lattices described in the next theorem we do not know whether there can be a measurable almost sure Steinhaus set.

Theorem 3. There exists a matrix $B \in G L(d, R)$ with the property that for any matrix $A$ if $A \prec B$, then $A \prec_{t} B$.

Proof of Theorem 3. Let the diagonal matrix $B$ have the form

$$
B=\left[\begin{array}{ccc}
\sqrt{\alpha_{1}} & & \\
& \cdots & \\
& & \sqrt{\alpha_{d}}
\end{array}\right],
$$

where $\alpha_{1}, \ldots, \alpha_{d}$ are independent over $\mathbb{Q}$. Suppose also that $A \prec B$. We express $A$ as a quadratic form:

$$
\|A \vec{x}\|^{2}=f(\vec{x})=\sum_{i=1}^{d} a_{i, i} x_{i}^{2}+\sum_{i=1}^{d} \sum_{j=i+1}^{d} a_{i, j} x_{i} x_{j} .
$$

From norm domination, we know that for any $\vec{x} \in \mathbb{Z}^{d}, f(\vec{x})=\alpha_{1}{\hat{x_{1}}}^{2}+\ldots+\alpha_{d}{\hat{x_{d}}}^{2}$ for some $\overrightarrow{\hat{x}} \in \mathbb{Z}^{d}$. It is clear that each of the $a_{i, j}$ are in the rational span of $\left\{\alpha_{1}, \ldots, \alpha_{d}\right\}$. Thus, we may write $a_{i, j}=a_{i, j, 1} \alpha_{1}+\ldots+a_{i, j, d} \alpha_{d}$, where each of the $a_{i, j, k}$ are rational. Thus, we may write

$$
\begin{aligned}
& f(\vec{x})=\alpha_{1}\left(\sum_{i=1}^{d} a_{i, i, 1} x_{i}^{2}+\sum_{i=1}^{d} \sum_{j=i+1}^{d} a_{i, j, 1} x_{i} x_{j}\right) \\
& +\ldots+\alpha_{d}\left(\sum_{i=1}^{d} a_{i, i, d} x_{i}^{2}+\sum_{i=1}^{d} \sum_{j=i+1}^{d} a_{i, j, d} x_{i} x_{j}\right) .
\end{aligned}
$$

We denote the components of this representation as

$$
f(\vec{x})=\alpha_{1} f_{1}(\vec{x})+\ldots+\alpha_{d} f_{d}(\vec{x}) .
$$

Since the $f_{i}$ are rational valued, since $f(\vec{x})=\alpha_{1}{\hat{x_{1}}}^{2}+\ldots+\alpha_{d}{\hat{x_{d}}}^{2}$ for some $\overrightarrow{\hat{x}} \in \mathbb{Z}^{d}$, and since $\alpha_{1}, \ldots, \alpha_{d}$ are independent over $\mathbb{Q}$, then the $f_{i}$ are always integer square valued. Here we state a lemma to be proven later.

Lemma 4. Any quadratic form which is always integer square valued is the square of an integer linear form.

This means that the $f_{i}$ are squares of linear forms. We may then write

$$
\begin{gathered}
f(\vec{x})=\alpha_{1}\left( \pm \sqrt{a_{1,1,1}} x_{1}+\ldots \pm \sqrt{a_{d, d, 1}} x_{d}\right)^{2} \\
\quad+\ldots+\alpha_{d}\left( \pm \sqrt{a_{1,1, d}} x_{1}+\ldots \pm \sqrt{a_{d, d, d}} x_{d}\right)^{2} \\
\|\|\left[\begin{array}{llll} 
\pm \sqrt{\alpha_{1} a_{1,1,1}} & \pm \sqrt{\alpha_{1} a_{2,2,1}} & \ldots & \pm \sqrt{\alpha_{1} a_{d, d, 1}} \\
\pm \sqrt{\alpha_{2} a_{1,1,2}} & \pm \sqrt{\alpha_{2} a_{2,2,2}} & \ldots & \pm \sqrt{\alpha_{2} a_{d, d, 2}} \\
\ldots & \ldots & \ldots & \ldots \\
\pm \sqrt{\alpha_{d} a_{1,1, d}} & \pm \sqrt{\alpha_{d} a_{2,2, d}} & \ldots & \pm \sqrt{\alpha_{d} a_{d, d, d}}
\end{array}\right] \|^{2}
\end{gathered}
$$


We can see that the $\sqrt{a_{i, i, j}}$ are integers, which gives us that the determinant of the above matrix is $\sqrt{\alpha_{1} \ldots \alpha_{d}}$ times the determinant of an integer matrix. Since this must also be the determinant of $A$, we have $\operatorname{det}(A) / \operatorname{det}(B)$ is an integer.

We now prove Lemma 4.

Proof of Lemma 4. We prove by induction on $d$, the number of variables in our quadratic form, beginning with our base case of $d=2$.

Suppose $f(x, y)=a x^{2}+b x y+c y^{2}$ is integer square valued on $\mathbb{Z}^{2}$. Our goal is to show that this is a linear form or, equivalently, to show that $b^{2}-4 a c=\Delta=0$. Assume, by way of contradiction that $\Delta \neq 0$. Considering $f(1,0)$ gives that $a$ is a square. We assume $a \neq 0$, as the alternative case is trivial. We have that $f(-b, 2 a)=a(-\Delta)$ is a square, and since $a$ is a nonzero square, we have $-\Delta$ is a square. We also have that $f(2 a \Delta-b, 2 a)=-\Delta a\left(4 a^{2}(-\Delta)+1\right)$ is a square. Since $-\Delta a$ is a nonzero square, we then have $\left(4 a^{2}(-\Delta)+1\right)$ is a square. On the other hand, we have that $4 a^{2}(-\Delta)$ is a square. This gives us two squares whose difference is one, which implies that $4 a^{2}(-\Delta)=0$, which yields a contradiction.

We now assume that any quadratic form in $n-1$ variables which is integer square valued on $\mathbb{Z}^{n-1}$ is the square of a linear form, and show the case of $n$. Assume that $f$ is integer square valued, where $f$ is given by:

$$
f\left(x_{1}, \ldots, x_{n}\right)=\sum_{i=1}^{n} a_{i, i} x_{i}^{2}+\sum_{i=1}^{n-1} n \sum_{j=i+1}^{d} a_{i, j} x_{i} x_{j} .
$$

By considering the case of all but two of the $x_{i}$ are equal to zero and applying the case of $d=2$, we find that each of the $a_{i, j}= \pm 2 \sqrt{a_{i, i} a_{j, j}}$. We now write $f$ as

$$
f\left(x_{1}, \ldots, x_{n}\right)=\left(\sum_{i=1}^{n-1} a_{i, i} x_{i}^{2}+\sum_{i=1}^{n-2} n \sum_{j=i+1}^{d-1} a_{i, j} x_{i} x_{j}\right)+\sum_{i=1}^{n} a_{i, n} x_{i} x_{n} .
$$

The parenthetical of the above is $f\left(x_{1}, \ldots, x_{n}-1,0\right)$, which is a binary form on $n-1$ variables, and which also is integer square valued. We apply our induction hypothesis to write:

$$
f\left(x_{1}, \ldots, x_{n}\right)=\left(\sum_{i=1}^{n-1} b_{i} x_{i}\right)^{2}+2 \sqrt{a_{n, n}} x_{n}\left(\sum_{i=1}^{n-1} c_{i} x_{i}\right)+a_{n, n} x_{n}^{2},
$$

where each of the $b_{i}= \pm \sqrt{a_{i, i}}$ and each of the $c_{i}= \pm \sqrt{a_{i, i}}$.

Now, if for all $i$ we have $b_{i}=c_{i}$, or if for all $i$ we have $b_{i}=-c_{i}$, then the above is the square of a linear form, and we are done. Suppose then, by way of contradiction, that $b_{j}=c_{j} \neq 0$, for some $j$, and $b_{k}=-c_{k} \neq 0$, for some $k \neq j$. Now consider the case of $x_{j}=b_{k} t, x_{k}=b_{j} t$, and $x_{i}=0$ for all $i$ not equal to $j$ or $k$. The above then reduces to

$$
=4 b_{j}^{2} b_{k}^{2} t^{2}+a_{n, n} x_{n}^{2},
$$

which is a binary quadratic form on $t$ and $x_{n}$ and, again, remains square valued. The base case gives us that $\Delta=-16 b_{j}^{2} b_{d}^{2} a_{n, n}=0$. We have assumed $b_{j} \neq 0 \neq b_{k}$, so we must have $a_{n, n}=0$, which gives us that our form on $n$ variables is actually a form on $n-1$ variables. Our induction hypothesis then completes the proof.

This result and the result of the previous section seem to indicate that the ability of a matrix $A$ to weakly norm dominate any matrix is related to the dimension of the 
entries of $A^{T} A$ in the rationals. A reasonable conjecture might be that, for $d=3$, $A$ weakly norm dominates another matrix if and only if $A$ is a constant times a norm rational matrix. This conjecture cannot hold in general dimension, however, by a counterexample in dimension 6 . (Consider a diagonal matrix which has its diagonal comprised of two diagonal matrices from dimension 3 which do weakly norm dominate, one of which is rational, the other multiplied by a transcendental.)

\section{A honeycomb lattice: The Standard tetrahedral lattice}

Here we apply Theorem 1 to show that there is no measurable set with the Steinhaus property over the 3 -dimensional standard tetrahedral lattice. The vectors which generate this honeycomb lattice may be visualized by considering three edges of a regular tetrahedron which have a vertex in common. That is, they are three unit vectors, each pair of which has an angle of sixty degrees.

Theorem 5. There is no measurable set with the Steinhaus property over the honeycomb lattice $H$, where $H$ is given by:

$$
H=\left[\begin{array}{lll}
1 & \frac{1}{2} & \frac{1}{2} \\
& \frac{\sqrt{3}}{2} & \frac{\sqrt{3}}{6} \\
& & \frac{\sqrt{6}}{3}
\end{array}\right], \quad H^{-T}=\left[\begin{array}{llll}
1 & & \\
\frac{-1}{3} \sqrt{3} & \frac{2}{3} \sqrt{3} & \\
\frac{-1}{6} \sqrt{6} & \frac{-1}{6} \sqrt{6} & \frac{1}{2} \sqrt{6}
\end{array}\right] .
$$

Proof of Theorem 5. From Theorem 1, our goal is to find a matrix $A$, such that $A \prec_{w} H^{-T}$. To do this, we actually find two matrices $A$ and $B$, such that $A \prec_{s} B$ and $B \prec_{t} H^{-T}$. We begin by going through the steps of the proof of Theorem 2 to find $B$.

$$
\begin{gathered}
\mathcal{D}\left(H^{-T}\right)=\left\{x^{2}+\frac{1}{3}(-x+2 y)^{2}+\frac{1}{6}(-x-y+3 z)^{2}: \vec{x} \in \mathbb{Z}^{3}\right\} \\
\supseteq\left\{x_{1}^{2}(\hat{x})^{2}+\frac{4 y_{1}^{2}}{3}\left(\frac{-x_{1}}{2 y_{1}} \hat{x}+\hat{y}\right)^{2}+\frac{3 z_{1}}{2}\left(\frac{-x_{1}}{3 z_{1}} \hat{x}-\frac{y_{1}}{3 z_{1}} \hat{y}+\hat{z}\right)^{2}: \overrightarrow{\hat{x}} \in \mathbb{Z}^{3}\right\},
\end{gathered}
$$

if $x_{1}, y_{1}$, and $z_{1}$ are integers. We must choose $x_{1}, y_{1}$, and $z_{1}$ such that $\frac{-x_{1}}{2 y_{1}}, \frac{-x_{1}}{3 z_{1}}$, and $\frac{y_{1}}{3 z_{1}}$ are integers. The simplest such choice is $\left(x_{1}, y_{1}, z_{1}\right)=(6,3,1)$. This gives us

$$
\begin{gathered}
=\left\{36(\hat{x})^{2}+12(-\hat{x}+\hat{y})^{2}+\frac{3}{2}(-2 \hat{x}-\hat{y}+\hat{z})^{2}: \overrightarrow{\hat{x}} \in \mathbb{Z}^{3}\right\} \\
=\mathcal{D}\left(\left[\begin{array}{ccc}
6 & & \\
& 2 \sqrt{3} & \\
& & \frac{1}{2} \sqrt{6}
\end{array}\right]\right)=\frac{3}{2}\left\{24 x^{2}+8 y^{2}+z^{2}: \vec{x} \in \mathbb{Z}^{3}\right\} .
\end{gathered}
$$

We now have shown:

$$
H^{-T}=\left[\begin{array}{lll}
1 & & \\
\frac{-1}{3} \sqrt{3} & \frac{2}{3} \sqrt{3} & \\
\frac{-1}{6} \sqrt{6} & \frac{-1}{6} \sqrt{6} & \frac{1}{2} \sqrt{6}
\end{array}\right] \succ_{t}\left[\begin{array}{lll}
6 & & \\
& 2 \sqrt{3} & \\
& & \frac{1}{2} \sqrt{6}
\end{array}\right]=B .
$$

Now, we need a matrix $A$, such that $B \succ_{s} A$. From this we will have that $H^{-T} \succ_{s} A$ and the proof will be complete. Let us define $A$ as follows:

$$
A=\left[\begin{array}{ccc}
2 \sqrt{3} & & \\
& \frac{\sqrt{102}}{2} & \\
& & 6
\end{array}\right] \text {. }
$$


We have that $\operatorname{det}(A) / \operatorname{det}(B)=\sqrt{17} / 2$ is irrational. We need to show that $\mathcal{D}(A) \subseteq \mathcal{D}(B)$, or that

$$
\left\{12 x^{2}+\frac{51}{2} y^{2}+36 z^{2}: \vec{x} \in \mathbb{Z}^{3}\right\} \subseteq\left\{36 x^{2}+12 y^{2}+\frac{3}{2} z^{2}: \vec{x} \in \mathbb{Z}^{3}\right\} .
$$

Multiply both sides by $\frac{2}{3}$ :

$$
\left\{8 x^{2}+17 y^{2}+24 z^{2}: \vec{x} \in \mathbb{Z}^{3}\right\} \subseteq\left\{24 x^{2}+8 y^{2}+z^{2}: \vec{x} \in \mathbb{Z}^{3}\right\} .
$$

It has been shown (see [6]) that those positive integers which cannot be expressed as $24 x^{2}+8 y^{2}+z^{2}$ are exactly those integers of the form $4 n+2,4 n+3$, or $4^{k}(8 n+5)$. By way of contradiction, let us suppose that $8 x^{2}+17 y^{2}+24 z^{2}=4 n+2,4 n+3$, or $4^{k}(8 n+5)$. We can immediately rule out $4 n+2$ and $4 n+3$ as we have $8 x^{2}+$ $17 y^{2}+24 z^{2} \equiv y^{2}(\bmod 4)$, and the quadratic residues $\bmod 4$ are 0 and 1 .

We consider the remaining case $8 x^{2}+17 y^{2}+24 z^{2}=4^{k}(8 n+5)$. We consider the values of $k$.

$k=0$ : Taking the equation $\bmod 8$ gives $y^{2} \equiv 5(\bmod 8)$, which is a contradiction, as the only squares mod 8 are 0,1 , and 4 .

$k=1:$ We have $8 x^{2}+17 y^{2}+24 z^{2}=4(8 n+5)$. Since the left side is then even, $y$ must be even. Write $y=2 y_{1}$. Then, dividing by 4 gives $2 x^{2}+y_{1}^{2}+6 z^{2} \equiv 5(\bmod$ 8 ). The only squares mod 8 are 0,1 , and 4 , and checking all cases shows that this is a contradiction.

$k \geq 2$ : We have $8 x^{2}+17 y^{2}+24 z^{2}=4^{k}(8 n+5)$. We must have $y$ divisible by 4 for the left side to be divisible by 8 , and we write $y=4 y_{1}$. This gives $x^{2}+$ $34 y_{1}^{2}+3 z^{2}=4^{k-2}(16 n+10)$. We see that $x$ and $z$ are both odd, or both even. If they are both even, then we write $x=2 x_{1}, z=2 z_{1}$, and $y=2 y_{2}$ and arrive at $8 x_{1}^{2}+17 y_{2}^{2}+24 z_{1}^{2}=4^{k-1}(8 n+5)$, from which we may repeat the argument and descend until $k<2$ or $x, z$ are odd.

Assume then that $x$ and $z$ are odd. We write $x=2 x_{1}+1, z=2 z_{1}+1$. This gives us $2 x_{1}\left(x_{1}+1\right)+17 y_{1}^{2}+6 z_{1}\left(z_{1}+1\right)+2=4^{k-2}(8 n+5)$. If $k>2$, then $y_{1}$ is even, which gives a contradiction, as 4 divides the right side, but not the left. So, assume $k=2$. We then have $2 x_{1}\left(x_{1}+1\right)+y_{1}^{2}+6 z_{1}\left(z_{1}+1\right) \equiv 3(\bmod 8)$. For the left side to be odd, we must have $y_{1}$ odd, which gives $y_{1}^{2} \equiv 1$, giving $2 x_{1}\left(x_{1}+1\right)+6 z_{1}\left(z_{1}+1\right) \equiv 2(\bmod$ $8)$. This yields $x_{1}\left(x_{1}+1\right)+3 z_{1}\left(z_{1}+1\right) \equiv 1(\bmod 4)$, which gives a contradiction, as the left side is even and the right is odd.

We now have $A \prec_{s} B$ and $B \prec_{t} H^{-T}$, which gives $A \prec_{w} H^{-T}$, which, by Theorem 1, completes the proof.

It is important to note that, in the above proof, we relied heavily on having a simple expression for those integers not of the form $x^{2}+8 y^{2}+24 z^{2}$. In [6], a ternary quadratic form of the form $a x^{2}+b y^{2}+c z^{2}$ is called regular if the set of positive integers not represented by it can be written as a union of arithmetic sequences. It is stated that there are exactly 102 regular forms when $\operatorname{gcd}(a, b, c)=1$, which indicates that proofs like this one will not apply to general $B$.

One of the simplest irregular forms is $x^{2}+y^{2}+7 z^{2}$. There is empirical evidence to suggest that

$$
\left\{x^{2}+8 y^{2}+28 z^{2}: \vec{x} \in \mathbb{Z}^{3}\right\} \subset\left\{x^{2}+y^{2}+7 z^{2}: \vec{x} \in \mathbb{Z}^{3}\right\} .
$$


Specifically, the subset relation holds when the images are restricted to the first 2000 integers. This would give an example of strong norm domination of an irregular form by an irregular form, but no means of proving that the subset holds in general is obvious to us.

\section{REFERENCES}

[1] J. Beck, On a lattice point problem of H. Steinhaus, Studia Sci. Math. Hung. 24 (1989), 263-268. MR 91i:11072

[2] H. T. Croft, Three lattice-point problems of Steinhaus, Quart. J. Math. 33 (1982), 71-82. MR 85g:11051

[3] M. N. Kolountzakis and M. Papadimitrakis, The Steinhaus tiling problem and the range of certain quadratic forms, to appear.

[4] Steve Jackson and R. Daniel Mauldin, On a lattice problem of Steinhaus, Jour. Amer. Math. Soc. 15 (2002), 817-856.

[5] M. N. Kolountzakis and T. Wolff, On the Steinhaus tiling problem, Mathematika 46 (1999), 253-280. MR 2002c:52024

[6] Leonard Eugene Dickson, Modern Elementary Theory of Numbers, The University of Chicago Press, Chicago, 1939 (see pp. 109-113). MR 1:65a

Department of Mathematics, Box 311430, University of North Texas, Denton, Texas 76203

E-mail address: mauldin@unt.edu

$U R L$ : www.math.unt.edu/ ${ }^{\sim m a u l d i n}$

Department of Mathematics, Box 311430, University of North Texas, Denton, Texas 76203

E-mail address: andyq@unt.edu 